and 46 , this quantity stabilizes at a nearly constant value for $I \geqslant 48$. Swiateckit describes this behaviour as a "hint of centrifugal solidification" at the largest angular momenta. That is, at these angular momenta the nucleus stretches with the nucleons remaining in their original states rather than being redistributed adiabatically into the lowest energy state.

${ }^{152} \mathrm{Dy}$ nuclei with angular momentum $I>28$ seem to prefer being in the superdeformed state, but below this the $\gamma$-ray transitions lead predominantly to the normal deformed state. By $I=24$ the superdeformed states are almost completely vacant. No calculations predict such an abrupt disappearance of the stable superdeformed shape. This feature may be associated ${ }^{8}$ with pairing, another property of nuclei. Because of the strong nuclear force and the quantum-mechanical Pauli principle, nucleons prefer to arrange themselves two by two in orbits with the partners moving in opposite directions. The consequences of nuclear pairing include nuclear superfluidity, the quasiparticle spectrum of nuclear states and special selection rules for pair transfer.

The decay from super to normal deformation can be considered as a penetration, enhanced by the pairing ${ }^{9,10}$, of the potential-energy barrier that separates the two structures. Coriolis and centrifugal forces in a rotating nucleus are predicted" and have been observed ${ }^{12}$ to quench pair correlations, just as the magnetic fields quench electron pairing in supercon- ductors. The de-population of the superdeformed configuration at $I<28$ can then be attributed ${ }^{4}$ to the onset of pair correlations, with its associated enhanced barrier penetration.

Investigations of high-spin superdeformation have begun at nearly all suitable facilities, but no other similarly deformed nuclei have yet been reported, although rumours abound. Deformations intermediate to normal deformed nuclei and superdeformed ${ }^{152} \mathrm{Dy}$, however, have been reported for cerium ${ }^{13}$, neodymium ${ }^{1.15}$, praseodymium $^{15}$, and zirconium ${ }^{16}$ isotopes, yielding further information about the shell structure in rotating deformed nuclei. The idea that added stability applies only for $a / b \sim 1.25$ (normal deformation) and 2 (superdeformation) is apparently too simple.

1. Twin, P.J. et al. Phys. Rev. Lett. 57. 811-814 (1986).

2. Garrett, J.D. Nature 323, 395 (1986)

3. Bentley, M.A. et al. Phys. Rev. Lett. (in the press)

4. Swiatecki, W. J. Phys, Rev, Lett, 58, 1184-1187 (1987).

5. Strutinsky. V.M. Nucl. Phys. A95. 420-442 (1967).

6. Polikanov, S.M. et al. J. exp. theor. Phys. 42, 1016-102 (1962).

7. Strutinsky, V.M. Nucl. Phys. A122, 1-33 (1968)

8. Cohen, S. et al. Ann. Phys. 82, 557-596 (1974).

9. Herskind, B. et al. NBI-Milano-Sevilla-Strasbourg preprint (1987)

10. Bertsch, G.F. Phys, Lett, 95B, 157-159 (1980).

11. Mottelson, B. \& Valatin, J.G. Phys. Rev. Lett. 5. 511-512 (1960)

12. Bacelar, J.C. et al. Nucl. Phys. A442, 509-546 (1985)

13. Kirwan, A.J. et al. Phys. Rev. Lett. 58, 467-470 (1987)

14. Beck. E.M. et al. Phys. Rev. Lett. 58. 2182-2185 (1987)

15. Love. D.J.G. et al. Proc. Int. Conf. Nuclear Shapes (Crete) 1, $56(1987)$.

16. Dudek, J. et al. Phys. Rev. C35. 1489-1501 (1987).

J.D. Garrett is at the Niels Bohr Institute, University of Copenhagen, DK-2100 Copenhagen, Denmark.

\title{
Biological diversity
}

\section{What makes a forest rich?}

\section{Peter D. Moore}

As soon as biologists became involved in voyages of discovery in the eighteenth and nineteenth centuries, they were struck by the global patterns of vegetation and animal communities and the ways in which some areas can support a greater diversity of life than others. This relates directly to the number of organisms that can make a living out of the available resources, which in turn may be dependent on the extent of these resources, the limitations of the physical environment, the ways in which resources present themselves for exploitation (habitat heterogeneity), the number of species geographically available, site accessibility, and so on. There have been many attempts to find out which factors are most important in producing species richness, but quantitative analysis has been difficult. Elsewhere in this issue (Nature 329, 326-327; 1987), D.J Currie and V. Paquin report their analysis of trees throughout North America, and identify the most influential variable that determines the species richness of forests there.
In the past, most research has been concentrated on the influence of latitude and isolation in controlling biological diversity, together with the effects of major climate and environmental disturbances such as the onset of glaciation. There is evidence for a general decline in richness of species from the tropics to the tundra, creating an overall latitudinal gradient in diversity; isolated locations (from peninsulas to islands) also exhibit declining species richness. In Europe, the depauperate state of the tree flora has been related to the generally east-west arrangement of the main mountain systems of this continent, the Pyrenees and Alps; these cut off the migration routes of tree species moving south as climates cooled and may have led to the extinction of some taxa that survived in the New World because of the generally north-south alignment of mountain chains there (the Rockies and the Appalacians).

However, this picture is complicated by factors such as habitat diversity, for the requirements of more species can be satisfied if a site is topographically or ecologically variable. When investigating frogs in Amazonia, B.L. Zimmerman and R.O. Bierregaard (J. Biogeogr. 13, 133$143 ; 1986)$, found the richest sites to be those which contained the mud wallows of the collared peccary, thus supplying the precise needs of breeding frogs. So generalizations about the causes of diversity may well be difficult to construct.

There is also the practical problem of taxonomy. Almost all studies of species richness concentrate, very naturally, on just one taxonomic group - frogs, lizards, birds, bats, flowering plants, and so on. But each group, like the frogs mentioned above, may have some peculiar requirement of its own that obscures the general causes of diversity. In the case of the study by Currie and Paquin reported in this issue, the raw data relate to trees. Dividing the whole of the United States and Canada into 336 quadrats, these authors map the occurrence of all 620 native tree species and draw contours of richness (see their Fig. 1 on page 326). It is immediately obvious that the tree data do not follow any simple pattern. There are no strong latitudinal gradients, especially in the west, and the areas with the highest richness are clearly concentrated in the southeast. The results do not tally with bird data, for rich and poor areas do not coincide, nor are trees noticeably less rich on peninsulas.

Currie and Paquin tested their correlations with several variables using nonlinear regression, and find the strongest relationship is between richness and annual evapotranspiration. For trees, it seems, dry spots are bad news. Once the authors allowed for this factor, high richness turned out to be associated with quadrats in which altitudinal variability is high - the habitat diversity effect again.

On a global scale, evapotranspiration is a major factor influencing primary productivity, so the results of Currie and Paquin imply that the richness of forest trees is essentially determined by potential productivity of an area. A further implication is that historical factors, such as the intensity or frequency of glaciation and problems of migration and immigration, are of lesser significance in controlling contemporary richness. By using their climatic model to predict the forest richness of the British Isles (and coming very close to the mark) Currie and Paquin show that Europeans cannot use their unfortunate experiences during the Pleistocene as an excuse for present-day botanical poverty; rather, it should be put down to climate. Forest richness, it seems, is mainly the product of the resource base - water, warmth and solar energy.

Peter D. Moore is Reader in Ecology at King's College London (KQC), Campden Hill Road, London W8 $7 A H$, UK. 